\title{
Transcendental entire functions of finite order sharing two sets of small functions with their shift differential operators
}

\author{
Abhijit Banerjee(D), Arpita Roy*(D) \\ Department of Mathematics, University of Kalyani, West Bengal 741235, India
}

\begin{abstract}
Dealing with a question initiated by Liu [Meromorphic functions sharing a set with applications to difference equations, J. Math. Anal. Appl., 2009], we have investigated the situation when a finite order entire function and its shift differential operator share two sets of small functions. Our result has improved and extended the results of Chen-Chen [Entire functions sharing sets of small functions with their difference operators or shifts, Math. Slovaca, 2013] and Cui-Chen [The conjecture on unity of meromorphic functions concerning their differences, J. Difference Equ. Appl., 2016]. We have exhibited several examples relevant to the content of the paper.
\end{abstract}

Mathematics Subject Classification (2020). 30D35, 39A70

Keywords. entire function, small function, uniqueness, reduced linear $c$-shift operator, finite order

\section{Introduction and results}

In this paper, the term "meromorphic" will be used to mean meromorphic in the whole complex plane, unless specifically stated otherwise. At the outset, we have assumed that the readers are familiar with the standard definitions and notations of the Nevanlinna theory. Still we suggest the readers to make a glance over $[5,8,9]$.

In addition, for any non-constant meromorphic function $f(z)$ and for all $r$ outside of a possible exceptional set of finite linear measure, we define $S(r, f)=o(T(r, f))$. We denote by $S(f)$ the set of all meromorphic functions $a(z)$ such that $T(r, a(z))=S(r, f)$ and $a(z)$ is called small function compared to $f(z)$.

Next we explain some definitions and notations which are used in the paper.

For some $a \in \mathbb{C}$, we denote by $E(a ; f)$, the collection of the zeros of $f-a$, where a zero is counted according to its multiplicity. In addition to this, when $a=\infty$, the above definition implies that we are considering the poles. In the same manner, by $\bar{E}(a ; f)$, we denote the collection of the distinct zeros or poles of $f-a$ according as $a \in \mathbb{C}$ or $a=\infty$ respectively. For any two non-constant meromorphic functions $f$ and $g$, if $E(a ; f)=E(a ; g)(\bar{E}(a ; f)=$ $\bar{E}(a ; g))$, we say that $f$ and $g$ share the value $a$ CM (IM). If $a=a(z)$ is a small function

*Corresponding Author.

Email addresses: abanerjee_kal@yahoo.co.in, abanerjeekal@gmail.com (A. Banerjee), arpita140793@gmail.com (A. Roy)

Received: 26.08.2020; Accepted: 14.06.2021 
we define that $f$ and $g$ share $a \mathrm{CM}$ or $a$ IM according as $f-a$ and $g-a$ share $0 \mathrm{CM}$ or 0 IM respectively.

Let $S_{1}$ be a subset containing distinct small functions of $S(f)$ and $E_{f}\left(S_{1}\right)=$ $\cup_{a(z) \in S_{1}}\{z: f(z)-a(z)=0\}$, where each zero is counted according to its multiplicity. If we do not count the multiplicity then the set $\cup_{a(z) \in S_{1}}\{z: f(z)-a(z)=0\}$ is denoted by $\bar{E}_{f}\left(S_{1}\right)$. If $E_{f}\left(S_{1}\right)=E_{g}\left(S_{1}\right)$ we say that $f$ and $g$ share the set $S_{1}$ CM. On the other hand, if $\bar{E}_{f}\left(S_{1}\right)=\bar{E}_{g}\left(S_{1}\right)$, we say that $f$ and $g$ share the set $S_{1}$ IM.

For a meromorphic function $f(z)$ and a non-zero complex constant $c$, the shift operator of $f(z)$ is denoted by $f(z+c)$. We use $\Delta_{c} f$ and $\Delta_{c}^{k} f$ to denote the difference and $k$-th order difference operators of $f(z)$, defined respectively by

$\Delta_{c} f=f(z+c)-f(z), \quad \Delta_{c}^{k} f=\Delta_{c}\left(\Delta_{c}^{k-1} f\right)=\sum_{i=0}^{k}(-1)^{k-i}\left(\begin{array}{c}k \\ i\end{array}\right) f(z+i c), \quad k \in \mathbb{N}, \quad k \geq 2$.

Next we define linear shift, shift-differential and differential operators respectively as follows:

$$
\begin{gathered}
L_{1}(f(z))=a_{0}(z) f(z)+\sum_{i=1}^{k} a_{i}(z) f\left(z+c_{i}\right), \\
L_{2}(f(z))=\sum_{i=1}^{s} b_{i}(z) f^{(i)}\left(z+c_{i}\right), \quad L_{3}(f(z))=\sum_{i=1}^{t} d_{i}(z) f^{(i)}(z),
\end{gathered}
$$

where $a_{i}(z)(i=0,1, \ldots, k) ; b_{i}(z)(i=1, \ldots, s) ; d_{i}(z)(i=1, \ldots, t) \in S(f)$ and all $c_{i}^{\prime} s$ are non-zero complex constants. For the sake of convenience we shall call $L_{1}(f(z))+L_{3}(f(z))$ as linear shift and differential operator and denote it by $\hat{L}(f(z))(\not \equiv 0)$. In particular, for some non-zero complex constant $c$ if we choose $c_{i}=i c(i=1, \ldots, k)$, and $a_{k}(z)$ $(\not \equiv 0)$ in $L_{1}(f(z))$ we call the operator as linear $c$-shift operator and is denoted by $L_{c} f=$ $\sum_{i=0}^{k} a_{i}(z) f(z+i c)$. In addition to it, if the coefficients of $L_{c} f$ are constants satisfying $\sum_{i=0}^{k} a_{i}=0$, then this operator will be called reduced linear $c$-shift operator and recently it has been denoted by $L_{c}^{r} f$ (see [1]). In this paper, for the first two theorems we use the operator $\hat{L}(f(z))$ while for the last one, we use the operator $L_{c}^{r} f$.

In 2009, Liu [7] first investigated the uniqueness of a finite order entire function together with its difference operator sharing a set containing two elements. Liu's [7] result was as follows:

Theorem 1.1. [7] Let $f$ be a transcendental entire function of finite order and let a be a non-zero finite constant. If $f$ and $\Delta_{c} f$ share the set $\{a,-a\} C M$, then $f(z+c) \equiv 2 f(z)$.

In [7] the author posed an open question: "What happens if in Theorem 1.1, $\{a,-a\}$ is replaced by $\{a(z), b(z)\}$ where $a(z), b(z) \in S(f)$ are two non-vanishing periodic functions with period $c$ ?"

Chen-Chen [2] answered the question of Liu [7] in the following way:

Theorem 1.2. [2] Let $f(z)$ be a transcendental entire function of finite order, $c \in \mathbb{C} \backslash\{0\}$ and let $a(z) \in S(f)$ is a periodic entire function with period $c$ such that $a(z) \not \equiv 0$. If $f$ and $L_{c} f$ share the sets $\{a(z),-a(z)\}$ and $\{0\} C M$, then $L_{c} f(z) \equiv \pm f(z)$ for all $z \in \mathbb{C}$.

In the paper, we have been able to reduce the sharing condition $\{0\} \mathrm{CM}$ in Theorem 1.2 to $\{0\}$ IM. We have also extended Theorem 1.2 for $\hat{L}(f(z))$ as follows: 
Theorem 1.3. Let $f(z)$ be a transcendental entire function of finite order, $\hat{L}(f(z))$ be the shift and differential operator such that $\hat{L}(f(z)) \not \equiv 0$ and $a(z)(\not \equiv 0) \in S(f)$ be an entire function. If $f(z)$ and $\hat{L}(f(z))$ share the sets $\{a(z),-a(z)\} C M$ and $\{0\}$ IM then $\hat{L}(f(z)) \equiv \pm f(z)$.

In the next theorem, we shall show that if sharing of CM, IM of the range sets in Theorem 1.3 is reversed the conclusion remains same.

Theorem 1.4. Under the same situation as in Theorem 1.3, if $f(z)$ and $\hat{L}(f(z))$ share the sets $\{a(z),-a(z)\} I M$ and $\{0\} C M$ then $\hat{L}(f(z)) \equiv \pm f(z)$.

Remark 1.5. Following the same procedure as used to prove the above two theorems, the same two can be extended for $L_{1}(f(z))+L_{2}(f(z))+L_{3}(f(z))$ also.

Following two examples show that in Theorems 1.3 and 1.4, the case $\hat{L}(f(z)) \equiv-f(z)$ actually occur.

Example 1.6. Let $f(z)=\sin z$. For a suitable choice of coefficients, one can make $\hat{L}(f(z))=-\sin z$. Then clearly $f$ and $\hat{L}(f(z))$ share the sets $\{1,-1\},\{0\} \mathrm{CM}$ and $\hat{L}(f(z))=-f(z)$.

Example 1.7. Let $f=\frac{e^{\frac{2 \pi i z}{c}}+1}{e^{\frac{\pi i z}{c}}}$. Choose $\hat{L}(f(z))=\frac{(-1)^{k-1}}{2^{k}} \Delta_{c}^{k} f=-f$. Clearly $f$ and $\hat{L}(f(z))$ share the sets $\{1,-1\},\{0\} \mathrm{CM}$ and $\hat{L}(f(z))=-f(z)$.

Next examples show that $\{0\}$ sharing can not be removed in Theorems 1.3 and 1.4.

Example 1.8. Let $f(z)=e^{2 z}+3 e^{z}+1$ and $\hat{L}(f(z))=a_{2} f\left(z+c_{2}\right)+a_{1} f\left(z+c_{1}\right)+a_{0} f(z)$. Choosing $c_{2}=\pi i ; c_{1}=\frac{\pi i}{2} ; a_{2}=\frac{11+15 i}{12} ; a_{1}=\frac{5}{2} ; a_{0}=\frac{43-15 i}{12}$, we get $\hat{L}(f(z))=2 e^{2 z}+$ $8 e^{z}+7$. One can easily check that $f$ and $\hat{L}(f(z))$ share the sets $\{1,-1\}$ IM but not share $\{0\}$ and so $\hat{L}(f(z)) \neq \pm f$.

Example 1.9. Let $f(z)=\sin z$. For a suitable choice of coefficients, one can make $\hat{L}(f(z))=\cos z$. e.g., choose $c_{1}=\frac{\pi}{2} ; a_{0}=d_{2}, a_{1}+d_{1}=1$ and all other coefficients are zero. Clearly we have $\hat{L}(f(z))=a_{0} f(z)+a_{1} f\left(z+c_{1}\right)+d_{1} f^{\prime}(z)+d_{2} f^{\prime \prime}(z)=\cos z$. Though $f$ and $\hat{L}(f(z))$ share the set $\left\{\frac{1}{\sqrt{2}},-\frac{1}{\sqrt{2}}\right\}$ CM but $\hat{L}(f(z)) \neq \pm f(z)$.

Following example shows that in Theorems 1.3 and 1.4, one can not replace two specific sets by two arbitrary sets.

Example 1.10. Let $f(z)=e^{\frac{\lambda z}{c}}+a+b$, where $e^{\lambda}-1=i$, where $a$ and $b$ be two distinct complex numbers. Clearly $\hat{L}(f(z))=\Delta_{c}^{k} f=i^{k} e^{\frac{\lambda z}{c}}$. For $k=4 m-2, m \in \mathbb{N}, \hat{L}(f(z))$ and $f$ share the sets $\{a, b\}$ and $\left\{\frac{a+b}{2}\right\} \mathrm{CM}$, but $\hat{L}(f(z)) \neq \pm f(z)$.

The following example shows that the infinite order entire function can also satisfy Theorems 1.3 and 1.4. However we were unable to deduce the analogous result of those two theorems for infinite order entire function.

Example 1.11. Let $f(z)=e^{-e^{\frac{\pi i z}{c}}}-e^{e^{\frac{\pi i z}{c}}}$. Then $\hat{L}(f(z))=\frac{1}{2^{k}} \Delta_{c}^{k} f=(-1)^{k} f(z)$. Here $\hat{L}(f(z))=(-1)^{k} f(z)$, which shows that $\hat{L}(f(z))= \pm f(z)$ according to $k$ is even or odd.

From the very definitions of sharing of sets we observe that the sharing of two sets $\{a(z),-a(z)\}$ and $\{0\}$ can also be visualized as a specific sharing of a single set comprises of three elements, namely, $\{a(z),-a(z), 0\}$. But the following example shows that Theorem 1.3 is not in general true for a set consisting of three elements for IM sharing.

Example 1.12. Following the same procedure as done in Example 1.9 we can find $\hat{L}(f(z))=\cos z$, when $f(z)=\sin z$. One can easily check that $f$ and $\hat{L}(f(z))$ share the set $\{1,-1,0\}$ IM but $\hat{L}(f(z)) \neq \pm f(z)$. 
In view of Example 1.12, the following question is inevitable:

Question 1.13. What happens, if under the same situations as in Theorem 1.3, $f$ and $\hat{L}(f(z))$ share a single set containing 3 elements?

By the following example, we see that a finite order meromorphic function $f$ and $\hat{L}(f(z))$ fulfill all the conditions of Theorems 1.3, 1.4 and $\hat{L}(f(z)) \equiv-f(z)$.

Example 1.14. Let $f(z)=\frac{e^{\frac{\pi i z}{c}}}{e^{\frac{2 \pi i z}{c}}+1}$. Then $\hat{L}(f(z))=\frac{1}{2} \Delta_{c} f=-\frac{e^{\frac{\pi i z}{c}}}{e^{\frac{2 \pi i z}{c}}+1}=-f$. Clearly $f$ and $\hat{L}(f(z))$ share the sets $\{a(z),-a(z)\}$ and $\{0\}$ CM.

Next we pose the following question:

Question 1.15. Are Theorems 1.3 and 1.4 valid for the operator $\Delta_{c} f$ or even for $\hat{L}(f(z))$, where $f$ is a finite order meromorphic function?

From Theorems 1.3 and 1.4, the following corollary immediately follows.

Corollary 1.16. In Theorems 1.3 and 1.4, particularly if $\hat{L}(f(z))$ is $\Delta_{c} f$ then $\Delta_{c} f \equiv f$.

In view of Theorem 1.3, it will be natural to investigate whether in the same theorem, the set $\{0\}$ can be replaced by $\{b(z)\}$, where $b(z)$ is non-zero periodic small function of $f$. In this respect, we would first like to mention the following contribution due to Chen-Chen [2].

Theorem 1.17. [2] Let $f(z)$ be a transcendental entire function of finite order, $c \in \mathbb{C} \backslash\{0\}$, and let $a(z)(\not \equiv 0), b(z) \in S(f)$ be two periodic entire functions with period $c$ such that $a(z)$ and $b(z)$ are linearly dependent over the complex field, but $b(z) \not \equiv \pm a(z)$. If $f(z)$ and $\Delta_{c} f$ share the sets $\{a(z),-a(z)\}$ and $\{b(z)\} C M$, and if the inequality

$$
N\left(r, \frac{1}{f(z)-b(z)}\right) \geq \lambda T(r, f),
$$

holds for $\lambda \in\left(\frac{2}{3}, 1\right]$, then

$$
\frac{\Delta_{c} f-b(z)}{f-b(z)}=t
$$

where $t \in \mathbb{C} \backslash\{0\}$.

In Theorem 1.17, as $t \in \mathbb{C} \backslash\{0\}$, the specific relation between the function and its difference operator can not be inferred directly. Considering constants in stead of small functions, in 2016, Cui-Chen [3] improved Theorem 1.17 as follows:

Theorem 1.18. [3] Let $f(z)$ be a transcendental entire function of finite order, and $c$ be a non-zero complex constant. Let $a$ and $b$ be two finite complex constants satisfying $b^{2}-a^{2} \neq 0$ and $n$ be a positive integer. If $\Delta_{c}^{n} f(z)$ and $f(z)$ share the sets $\{a,-a\}$ and $\{b\}$ $C M$, then $\Delta_{c}^{n} f(z) \equiv \pm f(z)$. Moreover if $b \neq 0$, then $\Delta_{c}^{n} f(z) \equiv f(z)$.

In view of Theorem 1.17, it will be interesting to explore the analogous result of Theorem 1.18 for small functions. In our next theorem, we are able to derive the straightforward relationship between the function and its reduced linear $c$-shift operator when they share two sets of small functions which improves Theorems 1.17 and 1.18.

Theorem 1.19. Let $f(z)$ be a transcendental entire function of finite order, $c$ be a complex number such that $L_{c}^{r} f \not \equiv 0$. Let $a(z)(\not \equiv 0), b(z)(\not \equiv 0) \in S(f)$ be two periodic small functions with period $c$ such that $b(z) \not \equiv \pm a(z)$. If $f$ and $L_{c}^{r} f$ share the sets $\{a(z),-a(z)\}$ $C M$ and $\{b(z)\} C M$, then $L_{c}^{r} f \equiv f$.

Remark 1.20. Putting $b=-a$ in Example 1.10, it is easy to see that in Theorem 1.19 the condition $b(z)(\not \equiv 0)$ is essential. 
In view of Theorem 1.19, it is quite natural to raise the question:

Question 1.21. Does Theorem 1.19 hold for $L_{1}(f(z))$ or even for $L_{1}(f(z))+L_{2}(f(z))+$ $L_{3}(f(z))$ sharing $\{a(z),-a(z)\} \mathrm{CM}$ and $\{b(z)\}$ IM?

Our next example shows that Theorems 1.3 and 1.4 are not true for any two finite order entire functions.

Example 1.22. Let $f(z)=e^{z}$ and $g(z)=e^{-z}$. Clearly $f$ and $g$ share the set $\{i,-i\}$ CM and $\{0\}$ CM but $g \not \equiv \pm f$.

\section{Lemmas}

Lemma 2.1. [4] Let $f(z)$ be a meromorphic function of finite order and $c \in \mathbb{C} \backslash\{0\}$. Then

$$
m\left(r, \frac{f(z+c)}{f(z)}\right)+m\left(r, \frac{f(z)}{f(z+c)}\right)=S(r, f) .
$$

Lemma 2.2. [9] Let $f(z)$ be a non-constant meromorphic function in the complex plane, and let $R(f)=\frac{P(f)}{Q(f)}$, where

$$
P(f)=\sum_{k=0}^{p} a_{k}(z) f^{k} \text { and } Q(f)=\sum_{j=0}^{q} b_{j}(z) f^{j}
$$

are two mutually prime polynomials in $f$. If the coefficients $a_{k}(z)$ for $k=0,1, \ldots, p$ and $b_{j}(z)$ for $j=0,1, \ldots, q$ are small functions of $f$ with $a_{p}(z) \not \equiv 0$ and $b_{q}(z) \not \equiv 0$, then

$$
T(r, R(f))=\max \{p, q\} T(r, f)+S(r, f) .
$$

Lemma 2.3. ([4]:Corollary 3.4 or [6]:Theorem 2.4) Let $w(z)$ be a non-constant finite order meromorphic solution of

$$
P(z, w)=0,
$$

where $P(z, w)$ is a difference polynomial in $w(z)$. If $P(z, a) \not \equiv 0$ for a meromorphic function $a(z)$ satisfying $T(r, a)=S(r, w)$, then

$$
m\left(r, \frac{1}{w-a(z)}\right)=S(r, w),
$$

where the exceptional set associated with $S(r, w)$ has at most finite logarithmic measure.

Lemma 2.4. Let $f(z)$ be a non-constant meromorphic function in $\mathbb{C}$ and $p \in \mathbb{C}$. Then for a small function $b(z)$ of $f$,

$$
m\left(r, \frac{\hat{L}(f(z))+p \hat{L}(b(z))}{f(z)+p b(z)}\right)=S(r, f) .
$$

When $\hat{L}(f(z))=L_{c}^{r} f(z)$ and $b(z)$ is a periodic small function of $f$ with period $c$, then

$$
m\left(r, \frac{L_{c}^{r} f(z)}{f(z)+p b(z)}\right)=S(r, f) .
$$

Proof. Clearly,

$$
\hat{L}(f(z)+p b(z))=\hat{L}(f(z))+p \hat{L}(b(z)) .
$$

Let $g(z)=f(z)+p b(z)$. Then $g$ is a meromorphic function with $T(r, g)=T(r, f)+S(r, f)$ and so $S(r, g)=S(r, f)$. 
Therefore in view of Lemma 2.1,

$$
\begin{aligned}
m\left(r, \frac{\hat{L}(f(z))+p \hat{L}(b(z))}{f(z)+p b(z)}\right) & =m\left(r, \frac{\hat{L}(f(z)+p b(z))}{f+p b(z)}\right) \\
& =m\left(r, \frac{\hat{L}(g(z))}{g(z)}\right)=S(r, g)=S(r, f) .
\end{aligned}
$$

Since $b(z)$ is a periodic small function of $f$ with period $c$, so from the definition of $L_{c}^{r} f$ we obtain $L_{c}^{r} b(z)=0$. Therefore,

$$
m\left(r, \frac{L_{c}^{r} f(z)}{f+p b(z)}\right)=S(r, f) .
$$

Lemma 2.5. Let $f(z)$ be a transcendental entire function of finite order, $c \in C \backslash\{0\}$, and let $a(z) \in S(f)$ be a periodic entire function with period $c$ such that $a(z) \not \equiv 0$. If $f(z)$ and $L_{c}^{r} f(z)$ share the sets $\{a(z),-a(z)\} C M$, then

$$
\left(L_{c}^{r} f-a(z)\right)\left(L_{c}^{r} f+a(z)\right)=e^{2 \gamma(z)}(f-a(z))(f+a(z)),
$$

where $\gamma(z)$ is a polynomial such that $T\left(r, e^{2 \gamma(z)}\right)=S(r, f)$.

Proof. Set $L_{c}^{r} f(z)=g(z)$ and $P(h)=a(z) h^{\prime}-a^{\prime}(z) h$ for some finite order entire function $h$. Clearly $g$ and $P(f)$ are entire functions and so $P(g)$ is also an entire function.

So, by Lemma 2.4 we have,

$$
T(r, g)=m(r, g) \leq m(r, f)+m\left(r, \frac{g}{f}\right)+O(1)=T(r, f)+S(r, f) .
$$
have

Therefore $g$ is of finite order. Since $f$ and $g$ share the sets $\{a(z),-a(z)\}$ CM, so we

$$
(g-a(z))(g+a(z))=e^{2 \gamma(z)}(f-a(z))(f+a(z)),
$$

where $\gamma(z)$ is a polynomial. Also, we have

$$
\begin{aligned}
N\left(r, \frac{1}{g-a(z)}\right)+N\left(r, \frac{1}{g+a(z)}\right) & =N\left(r, \frac{1}{(g-a(z))(g+a(z))}\right) \\
& =N\left(r, \frac{1}{(f-a(z))(f+a(z))}\right) \\
& =N\left(r, \frac{1}{f-a(z)}\right)+N\left(r, \frac{1}{f+a(z)}\right) .
\end{aligned}
$$

So, by the second main value theorem we can obtain,

$$
\begin{aligned}
T(r, f) & \leq \bar{N}\left(r, \frac{1}{f-a(z)}\right)+\bar{N}\left(r, \frac{1}{f+a(z)}\right) \\
& =\bar{N}\left(r, \frac{1}{g-a(z)}\right)+\bar{N}\left(r, \frac{1}{g+a(z)}\right) \\
& \leq 2 T(r, g)+S(r, f) .
\end{aligned}
$$

Therefore, from (2.1) and (2.4) we can get $S(r, f)=S(r, g)$. For convenience, let $S(r)=$ $S(r, f)=S(r, g)$. From (2.2) we get, $T(r, \gamma(z))=S(r)$.

Clearly $P(f) \neq 0$ as well as $P(g) \neq 0$. On the contrary suppose $P(f)=0$, then

$$
f^{\prime} a(z)-f a^{\prime}(z)=0 \text {. }
$$

Integrating we have,

$$
f(z)=A a(z)
$$


where $A$ is non-zero constant. This immediately follows that $T(r, f)=S(r, f)$. Similarly if suppose $P(g)=0$, then $T(r, g)=S(r, f)$. So by $(2.4), T(r, f)=S(r, f)$, which is not possible.

Note that,

$$
a(z)\left(h h^{\prime}-a(z) a^{\prime}(z)\right)=h P(h)+a^{\prime}(z)\left(h^{2}-a^{2}(z)\right) .
$$

By taking derivative in both sides of (2.2) we have,

$$
g g^{\prime}-a(z) a^{\prime}(z)=e^{2 \gamma}\left[\gamma^{\prime}\left(f^{2}-a^{2}(z)\right)+\left(f f^{\prime}-a(z) a^{\prime}(z)\right)\right] .
$$

Using (2.5) we can write,

$$
g P(g)+a^{\prime}(z)\left(g^{2}-a^{2}(z)\right)=e^{2 \gamma}\left[a(z) \gamma^{\prime}\left(f^{2}-a^{2}(z)\right)+f P(f)+a^{\prime}(z)\left(f^{2}-a^{2}(z)\right)\right] .
$$

Therefore by (2.2),

$$
g P(g)=e^{2 \gamma} f P(f)+a(z) \gamma^{\prime}\left(g^{2}-a^{2}(z)\right) .
$$

Let $z_{0}$ be a zero of $(f-a(z))(f+a(z))$. Since $f(z)$ and $L_{c}^{r} f(z)$ share the sets $\{a(z),-a(z)\}$ $\mathrm{CM}$, so $z_{0}$ is also a zero of $(g-a(z))(g+a(z))$. This implies $g\left(z_{0}\right)= \pm f\left(z_{0}\right)= \pm a\left(z_{0}\right)$. Then from (2.6) it is clear that $z_{0}$ is zero of $a^{2}(z)\left[\left(e^{2 \gamma} P(f)\right)^{2}-(P(g))^{2}\right]$. If $a\left(z_{0}\right) \neq 0$ then obviously $z_{0}$ is zero of $\left(e^{2 \gamma} P(f)\right)^{2}-(P(g))^{2}$. If $a\left(z_{0}\right)=0$, then $\left(f\left(z_{0}\right)-a\left(z_{0}\right)\right)\left(f\left(z_{0}\right)+a\left(z_{0}\right)\right)=0$ and $\left(g\left(z_{0}\right)-a\left(z_{0}\right)\right)\left(g\left(z_{0}\right)+a\left(z_{0}\right)\right)=0$ gives $f\left(z_{0}\right)=0=g\left(z_{0}\right)$ and then automatically $z_{0}$ is zero of $\left(e^{2 \gamma} P(f)\right)^{2}-(P(g))^{2}$. Therefore all zeros of $(g-a(z))(g+a(z))$ are zeros of $\left(e^{2 \gamma} P(f)\right)^{2}-(P(g))^{2}$. Suppose $z_{0}$ is a zero of $(g-a(z))(g+a(z))$ with multiplicity $k(\geq 2)$ such that $a\left(z_{0}\right) \neq 0$. As $P(f)=a(z) f^{\prime}-a^{\prime}(z) f=a(z)\left(f^{\prime}-a^{\prime}(z)\right)-a^{\prime}(z)(f-a(z))=$ $a(z)\left(f^{\prime}+a^{\prime}(z)\right)-a^{\prime}(z)(f+a(z))$ and similar expression holds for $P(g)$ also, we note that clearly $z_{0}$ is a zero of $P(f)$ and $P(g)$ with multiplicity $(k-1)$. Therefore, $z_{0}$ is a zero of $\left(e^{2 \gamma} P(f)\right)^{2}-(P(g))^{2}$ with multiplicity at least $2(k-1)$.

Let,

$$
\Phi=\frac{\left(e^{2 \gamma} P(f)-P(g)\right)\left(e^{2 \gamma} P(f)+P(g)\right)}{(g-a(z))(g+a(z))} .
$$

Hence $N(r, \Phi) \leq N\left(r, \frac{1}{a(z)}\right)=S(r)$.

Note that, for an entire function $h$ and for some complex constant $q$, we can deduce that

$$
\begin{aligned}
m\left(r, \frac{P(h)}{h+q a(z)}\right) & =m\left(r, \frac{h^{\prime} a(z)-h a^{\prime}(z)}{h+q a(z)}\right) \\
& =m\left(r, \frac{a(z)\left(h^{\prime}+q a^{\prime}(z)\right)}{h+q a(z)}-a^{\prime}(z)\right) \\
& =S(r, h)+S(r) .
\end{aligned}
$$

Using (2.2) we can write,

$$
\begin{aligned}
& \frac{e^{2 \gamma} P(f)-P(g)}{g-a(z)} \\
= & \frac{g P(f)}{(f-a(z))(f+a(z))}+\frac{a(z) P(f)}{(f-a(z))(f+a(z))}-\frac{P(g)}{g-a(z)} \\
= & \frac{g}{f-a(z)} \cdot \frac{P(f)}{f+a(z)}+\frac{1}{2}\left(\frac{P(f)}{f-a(z)}-\frac{P(f)}{f+a(z)}\right)-\frac{P(g)}{g-a(z)}
\end{aligned}
$$

and

$$
\begin{aligned}
& \frac{e^{2 \gamma} P(f)+P(g)}{g+a(z)} \\
= & \frac{g}{f-a(z)} \cdot \frac{P(f)}{f+a(z)}-\frac{1}{2}\left(\frac{P(f)}{f-a(z)}-\frac{P(f)}{f+a(z)}\right)+\frac{P(g)}{g+a(z)} .
\end{aligned}
$$


Applying Lemma 2.4 and (2.8), from (2.9) and (2.10) we have,

$$
m\left(r, \frac{e^{2 \gamma} P(f)-P(g)}{g-a(z)}\right)=S(r) \text { and } m\left(r, \frac{e^{2 \gamma} P(f)+P(g)}{g+a(z)}\right)=S(r) .
$$

Therefore from (2.7) we can get,

$$
T(r, \Phi)=m(r, \Phi)+N(r, \Phi)=S(r) .
$$

To go through the further process first of all we calculate some proximity functions which will be more useful for the next calculations.

Now, $T(r, P(f)) \leq T\left(r, f^{\prime}\right)+T(r, f)+S(r, f) \leq 2 T(r, f)+S(r, f)$. Similarly using $(2.1)$,

$$
T(r, P(g)) \leq T\left(r, g^{\prime}\right)+T(r, g)+S(r, f) \leq 2 T(r, g)+S(r, f) \leq 2 T(r, f)+S(r, f) .
$$

Therefore $S(r, P(f))$ and $S(r, P(g))$ can be replaced by $S(r, f)$.

As $P(g)=a(z) g^{\prime}-a^{\prime}(z) g=L_{c}^{r}(P(f))$, so it follows from Lemma 2.4 that

$$
m\left(r, \frac{P(g)}{P(f)}\right)=S(r, P(f))=S(r) .
$$

Also,

$$
\begin{gathered}
m\left(r, \frac{(P(f))^{\prime}}{P(f)}\right)=S(r, P(f))=S(r) \text { and } \\
m\left(r, \frac{(P(g))^{\prime}}{P(g)}\right)=S(r, P(g))=S(r) .
\end{gathered}
$$

If $\gamma(z)$ is constant, then automatically $T\left(r, e^{2 \gamma}\right)=S(r, f)$. So for a non-constant polynomial $\gamma(z)$, to prove $T\left(r, e^{2 \gamma}\right)=S(r, f)$ we now distinguish two cases.

Case 1. Suppose $\Phi \equiv 0$. Then from (2.7),

$$
e^{2 \gamma}= \pm \frac{P(g)}{P(f)}
$$

So by $(2.12)$, it follows that $T\left(r, e^{2 \gamma}\right)=S(r)$.

Case 2. Next suppose $\Phi \not \equiv 0$. Therefore, from (2.7) we have $e^{2 \gamma} P(f)-P(g) \not \equiv 0$ and $e^{2 \gamma} P(f)+P(g) \not \equiv 0$. Now by $(2.2)$ and $(2.7)$,

$$
\begin{aligned}
\frac{1}{e^{2 \gamma} P(f)-P(g)} & =\frac{e^{2 \gamma} P(f)+P(g)}{\Phi(g-a(z))(g+a(z))} \\
& =\frac{1}{\Phi}\left[\frac{P(f)}{(f-a(z))(f+a(z))}+\frac{P(g)}{(g-a(z))(g+a(z))}\right] \\
& =\frac{1}{2 a(z) \Phi}\left[\frac{P(f)}{f-a(z)}-\frac{P(f)}{f+a(z)}+\frac{P(g)}{g-a(z)}-\frac{P(g)}{g+a(z)}\right] .
\end{aligned}
$$

Using (2.8) and (2.11), it follows that

$$
m\left(r, \frac{1}{e^{2 \gamma} P(f)-P(g)}\right)=S(r) .
$$

In a similar manner we can get,

$$
m\left(r, \frac{1}{e^{2 \gamma} P(f)+P(g)}\right)=S(r) .
$$

Using above two results with (2.11), it follows from (2.7) that

$$
m\left(r, \frac{1}{(g-a(z))(g+a(z))}\right)=m\left(r, \frac{\Phi}{\left(e^{2 \gamma} P(f)-P(g)\right)\left(e^{2 \gamma} P(f)+P(g)\right)}\right)=S(r) .
$$


Therefore by Lemma 2.2,

$$
N\left(r, \frac{1}{(g-a(z))(g+a(z))}\right)=T\left(r, \frac{1}{(g-a(z))(g+a(z))}\right)=2 T(r, g)+S(r) .
$$

i.e.,

$$
N\left(r, \frac{1}{g-a(z)}\right)+N\left(r, \frac{1}{g+a(z)}\right)=2 T(r, g)+S(r) .
$$

Now we claim that

$$
N\left(r, \frac{1}{(g-a(z))(g+a(z))}\right)=\bar{N}\left(r, \frac{1}{(g-a(z))(g+a(z))}\right)+S(r) .
$$

Rewriting (2.6) with the help of (2.2) we get,

$$
\frac{g P(g)}{g^{2}-a^{2}(z)}=\frac{f P(f)}{f^{2}-a^{2}(z)}+a(z) \gamma^{\prime} .
$$

Also from (2.7) we have,

$$
\Phi\left(g^{2}-a^{2}(z)\right)=\left(e^{2 \gamma} P(f)\right)^{2}-(P(g))^{2},
$$

which in view of $(2.2)$ yields

$$
\frac{\Phi}{g^{2}-a^{2}(z)}=\left(\frac{P(f)}{f^{2}-a^{2}(z)}\right)^{2}-\left(\frac{P(g)}{g^{2}-a^{2}(z)}\right)^{2} .
$$

Applying (2.17) on the above equation we can obtain,

$$
\frac{g^{2} \Phi}{g^{2}-a^{2}(z)}=\left(\frac{g P(f)}{f^{2}-a^{2}(z)}\right)^{2}-\left(\frac{f P(f)}{f^{2}-a^{2}(z)}+a(z) \gamma^{\prime}\right)^{2} .
$$

i.e.,

$$
\frac{g^{2} \Phi}{g^{2}-a^{2}(z)}=\frac{\left(g^{2}-f^{2}\right)(P(f))^{2}}{\left(f^{2}-a^{2}(z)\right)^{2}}-a^{2}(z) \gamma^{\prime 2}-\frac{2 a(z) \gamma^{\prime} f P(f)}{f^{2}-a^{2}(z)} .
$$

Let $z_{0}$ be a zero of $(f-a(z))(f+a(z))$ with multiplicity $k(\geq 2)$ such that $a\left(z_{0}\right) \neq 0$. Since $f$ and $g$ share the set $\{a(z),-a(z)\}$ CM, so $z_{0}$ is a zero of $(g-a(z))(g+a(z))$ with the same multiplicity $k$. Since $g^{2}-f^{2}$ can be written as $\{(g+a(z))+(f-a(z))\}\{(g-a(z))-(f-a(z))\}$ or $\{(g+a(z))+(f-a(z))\}\{(g+a(z))-(f+a(z))\}$ or $\{(g-a(z))+(f+a(z))\}\{(g-$ $a(z))-(f-a(z))\}$ or $\{(g-a(z))+(f+a(z))\}\{(g+a(z))-(f+a(z))\}$, so it follows that $z_{0}$ is zero of $\frac{\left(g^{2}-f^{2}\right)(P(f))^{2}}{\left(f^{2}-a^{2}(z)\right)^{2}}$ of multiplicity at least $k+2(k-1)-2 k=k-2(\geq 0)$ that means not a pole. If $z_{0}$ is not a zero of $\gamma^{\prime}$, then $z_{0}$ is a simple pole of $\frac{2 a(z) \gamma^{\prime} f P(f)}{f^{2}-a^{2}(z)}$. Thus $z_{0}$ is a simple pole of right-hand side of $(2.18)$ and hence $z_{0}$ is a simple pole of $\frac{g^{2} \Phi}{g^{2}-a^{2}(z)}$ as long as $z_{0}$ is not a zero of $\Phi$. This contradicts the assumption that $z_{0}$ is a multiple zero of $(g-a(z))(g+a(z))$. Therefore, the above argument indicates that all zeros of $(g-a(z))(g+a(z))$ are simple as long as they are not zeros of $\Phi, \gamma^{\prime}$ and $a(z)$. Hence,

$$
\begin{aligned}
N\left(r, \frac{1}{(g-a(z))(g+a(z))}\right) \leq & \bar{N}\left(r, \frac{1}{(g-a(z))(g+a(z))}\right)+N\left(r, \frac{1}{a(z)}\right) \\
& +N\left(r, \frac{1}{\Phi}\right)+N\left(r, \frac{1}{\gamma \prime}\right) \\
\leq & \bar{N}\left(r, \frac{1}{(g-a(z))(g+a(z))}\right)+T(r, \gamma)+S(r) \\
\leq & N\left(r, \frac{1}{(g-a(z))(g+a(z))}\right)+S(r) .
\end{aligned}
$$


Therefore, our claim (2.16) is proved.

From (2.7),

$$
e^{4 \gamma}(P(f))^{2}=(P(g))^{2}+\Phi\left(g^{2}-a^{2}(z)\right)
$$

Differentiating both sides of above equation we have,

$2 e^{4 \gamma} P(f)\left[(P(f))^{\prime}+2 \gamma^{\prime} P(f)\right]=2 P(g)(P(g))^{\prime}+\Phi^{\prime}\left(g^{2}-a^{2}(z)\right)+2 \Phi\left(g g^{\prime}-a(z) a^{\prime}(z)\right)$.

Using (2.5) we have,

$$
\begin{aligned}
2 e^{4 \gamma} a(z) P(f)\left[(P(f))^{\prime}+2 \gamma^{\prime} P(f)\right]= & a(z)\left[2 P(g)(P(g))^{\prime}+\Phi^{\prime}\left(g^{2}-a^{2}(z)\right)\right] \\
& +2 \Phi\left[g P(g)+a^{\prime}(z)\left(g^{2}-a^{2}(z)\right)\right] .
\end{aligned}
$$

Eliminating $e^{4 \gamma}$ from (2.19) and (2.20) and using the fact $P(f) \neq 0$ we can obtain,

$$
\begin{aligned}
& 2 a(z)\left[(P(f))^{\prime}+2 \gamma^{\prime} P(f)\right]\left[(P(g))^{2}+\Phi\left(g^{2}-a^{2}(z)\right)\right] \\
& =a(z) P(f)\left[2 P(g)(P(g))^{\prime}+\Phi^{\prime}\left(g^{2}-a^{2}(z)\right)\right]+2 \Phi P(f)\left[g P(g)+a^{\prime}(z)\left(g^{2}-a^{2}(z)\right)\right] .
\end{aligned}
$$

i.e.,

$$
\begin{aligned}
& \left\{2 a(z) \Phi\left[(P(f))^{\prime}+2 \gamma^{\prime} P(f)\right]-a(z) \Phi^{\prime} P(f)-2 a^{\prime}(z) \Phi P(f)\right\}\left(g^{2}-a^{2}(z)\right) \\
& =2 P(g)\left[a(z) P(f)(P(g))^{\prime}+\Phi g P(f)-a(z) P(g)\left((P(f))^{\prime}+2 \gamma^{\prime} P(f)\right)\right] .
\end{aligned}
$$

Since all zeros of $(g-a(z))(g+a(z))$ are almost simple, so they can not be zero of $P(g)$. Using this fact we can say that all zeros of $(g-a(z))(g+a(z))$ are almost simple zeros of $a(z) P(f)(P(g))^{\prime}+\Phi g P(f)-a(z) P(g)\left((P(f))^{\prime}+2 \gamma^{\prime} P(f)\right)$.

Let

$$
\Phi_{1}=\frac{a(z) P(f)(P(g))^{\prime}+\Phi g P(f)-a(z) P(g)\left((P(f))^{\prime}+2 \gamma^{\prime} P(f)\right)}{(f-a(z))(f+a(z))} .
$$

From the above argument it is clear that $N\left(r, \Phi_{1}\right)=S(r)$. Now,

$$
\begin{aligned}
m\left(r, \Phi_{1}\right) \leq & 3 m\left(r, \frac{P(f)}{f-a(z)}\right)+4 m\left(r, \frac{P(f)}{f+a(z)}\right)+3 m\left(r, \frac{P(g)}{P(f)}\right)+m\left(r, \frac{(P(g))^{\prime}}{P(g)}\right) \\
& +m\left(r, \frac{g}{f-a(z)}\right)+m\left(r, \frac{(P(f))^{\prime}}{P(f)}\right)+m(r, \Phi)+S(r) .
\end{aligned}
$$

Applying Lemma 2.4 and (2.8), (2.11), (2.12), (2.13), (2.14) it is clear that $m\left(r, \Phi_{1}\right)=$ $S(r)$. Hence $T\left(r, \Phi_{1}\right)=S(r)$.

Subcase 2.1. Let $\Phi_{1} \not \equiv 0$. Then by Lemma 2.2 from (2.22) and then using (2.1), (2.8), (2.13), (2.14) we have,

$$
\begin{aligned}
& 2 T(r, f) \\
= & T\left(r, \Phi_{1}(f-a(z))(f+a(z))\right)+S(r) \\
= & m\left(r, \Phi_{1}(f-a(z))(f+a(z))\right)+S(r) \\
\leq & m(r, f g)+m\left(r, \frac{a(z) P(f)(P(g))^{\prime}+\Phi g P(f)-a(z) P(g)\left((P(f))^{\prime}+2 \gamma^{\prime} P(f)\right)}{f g}\right) \\
& +S(r) \\
\leq & T(r, f)+T(r, g)+4 m\left(r, \frac{P(f)}{f}\right)+3 m\left(r, \frac{P(g)}{g}\right)+m\left(r, \frac{(P(g))^{\prime}}{P(g)}\right) \\
& +m(r, \Phi)+m\left(r, \frac{(P(f))^{\prime}}{P(f)}\right)+S(r) \\
= & T(r, f)+T(r, g)+S(r) \leq 2 T(r, f)+S(r) .
\end{aligned}
$$

Therefore, $T(r, f)=T(r, g)+S(r)$. Thus, from (2.3) and (2.15),

$$
2 T(r, f)=2 T(r, g)+S(r)=N\left(r, \frac{1}{f-a(z)}\right)+N\left(r, \frac{1}{f+a(z)}\right)+S(r),
$$


which implies

$$
m\left(r, \frac{1}{f-a(z)}\right)+m\left(r, \frac{1}{f+a(z)}\right)=S(r)
$$

Hence from (2.2),

$$
\begin{aligned}
T\left(r, e^{2 \gamma}\right) \leq & m\left(r, \frac{g}{f-a(z)}\right)+m\left(r, \frac{g}{f+a(z)}\right)+m\left(r, \frac{1}{f-a(z)}\right) \\
& +m\left(r, \frac{1}{f+a(z)}\right)+S(r) .
\end{aligned}
$$

Applying Lemma 2.4, it follows from $(2.23)$ that $T\left(r, e^{2 \gamma}\right)=S(r)$.

Subcase 2.2. Let $\Phi_{1} \equiv 0$. Then from (2.21) and (2.22) we have,

$$
2 a(z) \Phi\left[(P(f))^{\prime}+2 \gamma^{\prime} P(f)\right]-a(z) \Phi^{\prime} P(f)-2 a^{\prime}(z) \Phi P(f)=0 .
$$

i.e.,

$$
\frac{\Phi^{\prime}}{\Phi}=2 \frac{(P(f))^{\prime}}{P(f)}+4 \gamma^{\prime}-2 \frac{a^{\prime}(z)}{a(z)} .
$$

Integrating in both sides we obtain,

$$
\left(e^{2 \gamma} P(f)\right)^{2}=A \Phi a^{2}(z),
$$

where $A(\neq 0)$ is a constant. Putting (2.24) in (2.7) and simplifying we have,

$$
(P(g))^{2}=-\Phi\left[g^{2}-(1+A) a^{2}(z)\right] .
$$

Now, our claim is $A \neq-1$. On the contrary suppose $A=-1$. Then from (2.24) and (2.25) we have,

$$
\Phi=-\left(\frac{e^{2 \gamma} P(f)}{a(z)}\right)^{2} \text { and } \Phi=-\left(\frac{P(g)}{g}\right)^{2} .
$$

i.e.,

$$
\left(\frac{e^{2 \gamma} P(f)}{a(z)}\right)^{2}=\left(\frac{P(g)}{g}\right)^{2},
$$

which implies that

$$
e^{2 \gamma}= \pm \frac{a(z) P(g)}{g P(f)}
$$

Clearly, first equation of $(2.26)$ shows that $N\left(r, \frac{1}{P(f)}\right) \leq N\left(r, \frac{1}{a(z)}\right)+N\left(r, \frac{1}{\Phi}\right)=S(r)$. Therefore by (2.12), we have

$$
T\left(r, \frac{a(z) P(g)}{P(f)}\right)=N\left(r, \frac{a(z) P(g)}{P(f)}\right)+S(r) \leq N\left(r, \frac{1}{P(f)}\right)+S(r)=S(r) .
$$

Substituting (2.27) in (2.2) we get,

$$
g(g-a(z))(g+a(z))= \pm a(z) \frac{P(g)}{P(f)}(f-a(z))(f+a(z)) .
$$

Since $\frac{a(z) P(g)}{P(f)} \neq 0$ and $T\left(r, \frac{a(z) P(g)}{P(f)}\right)=S(r)$, so by Lemma 2.2 we can conclude that

$$
3 T(r, g)=2 T(r, f)+S(r) .
$$

Rewriting (2.6) we have,

$$
e^{2 \gamma} f P(f)=g^{2}\left(\frac{P(g)}{g}-a(z) \gamma^{\prime}\right)+a^{3}(z) \gamma^{\prime}
$$


Now, from (2.26) we have $T\left(r, e^{2 \gamma} P(f)\right)=S(r)$ and $T\left(r, \frac{P(g)}{g}\right)=S(r)$. Note that, $e^{2 \gamma} P(f) \neq 0$. Therefore if $\frac{P(g)}{g}-a(z) \gamma^{\prime} \neq 0$, then by Lemma 2.2 we can obtain that

$$
T(r, f)=2 T(r, g)+S(r),
$$

which together with (2.28) makes a contradiction $T(r, f)=S(r)$. Otherwise if $\frac{P(g)}{g}-a(z) \gamma^{\prime}=0$, then from (2.29) we have $e^{2 \gamma} f P(f)=a^{3}(z) \gamma^{\prime}$, which shows that $\stackrel{g}{T}(r, f)=S(r)$, a contradiction.

Hence our claim is proved.

Let $q^{2}=1+A$. So $q$ is a non-zero complex constant. Then rewriting $(2.25)$ we have,

$$
\begin{aligned}
\frac{1}{P(g)} & =-\frac{P(g)}{\Phi(g-q a(z))(g+q a(z))} \\
& =-\frac{1}{2 q a(z) \Phi}\left[\frac{P(g)}{g-q a(z)}-\frac{P(g)}{g+q a(z)}\right] .
\end{aligned}
$$

Therefore by (2.8) we can obtain, $m\left(r, \frac{1}{P(g)}\right)=S(r)$. This together with (2.8) and (2.12) produces that

$$
\begin{aligned}
m\left(r, \frac{1}{(f-a(z))(f+a(z))}\right) \leq & m\left(r, \frac{P(f)}{(f-a(z))(f+a(z))}\right)+m\left(r, \frac{P(g)}{P(f)}\right) \\
& +m\left(r, \frac{1}{P(g)}\right)+O(1) \\
\leq & m\left(r, \frac{P(f)}{f-a(z)}\right)+m\left(r, \frac{P(f)}{f+a(z)}\right)+S(r) \\
= & S(r) .
\end{aligned}
$$

Combining (2.2) and the above equation, by Lemma 2.4, we have

$$
\begin{aligned}
T\left(r, e^{2 \gamma}\right) & \leq m\left(r, \frac{g^{2}}{(f-a(z))(f+a(z))}\right)+m\left(r, \frac{1}{(f-a(z))(f+a(z))}\right)+S(r) \\
& \leq m\left(r, \frac{g}{f-a(z)}\right)+m\left(r, \frac{g}{f+a(z)}\right)+S(r) \\
& =S(r) .
\end{aligned}
$$

Hence the proof is completed.

\section{Proofs of the theorems}

Proof of Theorem 1.3. Using logarithmic derivative lemma and Lemma 2.1 we obtain that,

$$
\begin{aligned}
& T(r, \hat{L}(f(z)))=m(r, \hat{L}(f(z))) \\
= & m\left(r, a_{0}(z) f(z)+\sum_{i=1}^{k} a_{i}(z) f\left(z+c_{i}\right)+\sum_{j=1}^{t} d_{j}(z) f^{(j)}(z)\right) \\
\leq & m(r, f)+S(r, f) \leq T(r, f)+S(r, f),
\end{aligned}
$$

which gives $S(r, \hat{L}(f(z)))$ can be replaced by $S(r, f)$.

Let $g=\hat{L}(f(z))$. Suppose $g \not \equiv \pm f$. Take

$$
\alpha(z)=\frac{P(f)\left[g^{2}-f^{2}\right]}{f(f-a(z))(f+a(z))},
$$


where $P(f)$ has the same meaning as used in Lemma 2.5. Here $P(f) \not \equiv 0$. This already have been discussed in proof of Lemma 2.5. So, it is obvious that $\alpha(z) \not \equiv 0$. Now by Lemma 2.4 and (2.8), we obtain

$$
\begin{aligned}
m(r, \alpha(z)) & =m\left(r, \frac{P(f)\left[g^{2}-f^{2}\right]}{f(f-a(z))(f+a(z))}\right) \\
& \leq m\left(r, \frac{f P(f)}{(f-a(z))(f+a(z))}\right)+m\left(r, \frac{g^{2}}{f^{2}}-1\right)+O(1) \\
& \leq m\left(r, \frac{P(f)[(f-a(z))+(f+a(z))]}{2(f-a(z))(f+a(z))}\right)+S(r, f) \\
& \leq m\left(r, \frac{P(f)}{f-a(z)}\right)+m\left(r, \frac{P(f)}{f+a(z)}\right)+S(r, f) \\
& =S(r, f) .
\end{aligned}
$$

Let $z_{0}$ be a zero of $f$ with multiplicity $k_{1}$ such that $a\left(z_{0}\right) \neq 0$. Since $f$ and $g$ share the set $\{0\} \mathrm{IM}$, so $z_{0}$ is also a zero of $g$ with multiplicity $k_{2}$ (say). Then $z_{0}$ is zero of $\alpha(z)$ with multiplicity at least $2 \min \left\{k_{1}, k_{2}\right\}-1(\geq 1)$. So we can write

$$
\bar{N}\left(r, \frac{1}{f}\right) \leq \bar{N}\left(r, \frac{1}{\alpha(z)}\right)+\bar{N}\left(r, \frac{1}{a(z)}\right) \leq \bar{N}\left(r, \frac{1}{\alpha(z)}\right)+S(r, f) .
$$

Now, let $z_{1}$ be a zero of $(f-a(z))(f+a(z))$ with multiplicity $k$ such that $a\left(z_{1}\right) \neq 0$. Since $f$ and $g$ share the set $\{a(z),-a(z)\}$ CM, so $z_{1}$ is also a zero of $(g-a(z))(g+a(z))$ with the same multiplicity $k$. Then clearly $z_{1}$ is zero of $\alpha(z)$ with multiplicity at least $k-1$. Thus no zeros of $f$ and $(f-a(z))(f+a(z))$ are poles of $\alpha(z)$ as long as they are not zeros of $a(z)$. So we have,

$$
N(r, \alpha(z)) \leq N\left(r, \frac{1}{a(z)}\right)=S(r, f) .
$$

Therefore by (3.2) and (3.4) we get,

$$
T(r, \alpha(z))=S(r, f) .
$$

So from (3.3) and (3.5) we have,

$$
\bar{N}\left(r, \frac{1}{f}\right)=S(r, f) .
$$

By the Second Fundamental Theorem, it follows that

$$
\begin{aligned}
T\left(r, f^{2}\right) & \leq \bar{N}\left(r, f^{2}\right)+\bar{N}\left(r, \frac{1}{f^{2}}\right)+\bar{N}\left(r, \frac{1}{f^{2}-a^{2}(z)}\right)+S(r, f) \\
& \leq \bar{N}\left(r, \frac{1}{f^{2}-a^{2}(z)}\right)+S(r, f) \\
& \leq T\left(r, f^{2}\right)+S(r, f) .
\end{aligned}
$$

i.e.,

$$
N\left(r, \frac{1}{f^{2}-a^{2}(z)}\right)=\bar{N}\left(r, \frac{1}{f^{2}-a^{2}(z)}\right)=T\left(r, f^{2}\right)+S(r, f) .
$$

Therefore by the First Fundamental Theorem, $m\left(r, \frac{1}{f^{2}-a^{2}(z)}\right)=S(r, f)$. Clearly in view of (3.1), $g$ is of finite order as $f$ is so. As $f, g$ are of finite order and share the sets $\{a(z),-a(z)\}$ CM so,

$$
(g-a(z))(g+a(z))=e^{2 \gamma(z)}(f-a(z))(f+a(z)),
$$


where $\gamma(z)$ is a polynomial. Now, using Lemma 2.4 we get,

$$
\begin{aligned}
& T\left(r, e^{2 \gamma(z)}\right) \\
\leq & m\left(r, \frac{g^{2}-(\hat{L}(a(z)))^{2}}{(f-a(z))(f+a(z))}\right)+m\left(r, \frac{(\hat{L}(a(z)))^{2}-a^{2}(z)}{(f-a(z))(f+a(z))}\right)+S(r) \\
\leq & m\left(r, \frac{g-\hat{L}(a(z))}{f-a(z)}\right)+m\left(r, \frac{g+\hat{L}(a(z))}{f+a(z)}\right)+m\left(r, \frac{1}{(f-a(z))(f+a(z))}\right)+S(r) \\
= & S(r) .
\end{aligned}
$$

Let $d(z)=a^{2}(z)\left(1-e^{-2 \gamma(z)}\right)$. Since $g \not \equiv \pm f$, so $e^{2 \gamma(z)} \not \equiv 1$. So obviously $d(z) \not \equiv 0$. Rewriting (3.7) we get,

$$
g^{2}=e^{2 \gamma(z)}\left[f^{2}-d(z)\right]
$$

Therefore,

$$
\bar{N}\left(r, \frac{1}{g}\right)=\bar{N}\left(r, \frac{1}{f^{2}-d(z)}\right) .
$$

Since $f, g$ share 0 IM so from (3.6) we have,

$$
\bar{N}\left(r, \frac{1}{f^{2}-d(z)}\right)=\bar{N}\left(r, \frac{1}{g}\right)=\bar{N}\left(r, \frac{1}{f}\right)=S(r, f) .
$$

Hence by the Second Fundamental Theorem for small functions we deduce that

$$
T\left(r, f^{2}\right) \leq \bar{N}\left(r, \frac{1}{f^{2}}\right)+\bar{N}\left(r, \frac{1}{f^{2}-d(z)}\right)+S(r, f)=S(r, f) .
$$

Therefore we immediately get $g \equiv \pm f$, i.e., $\hat{L}(f(z)) \equiv f(z)$.

Proof of Theorem 1.4. Since $f$ and $\hat{L}(f(z))$ share the set $\{0\}$ CM, which implies $f$ and $\hat{L}(f(z))$ share the set $\{0\}$ IM. So proceeding in a similar manner as done in Theorem 1.3 , we can reach up to (3.3).

Now, let $z_{1}$ be a zero of $(f-a(z))(f+a(z))$ with multiplicity $k_{1}$ such that $a\left(z_{1}\right) \neq 0$. Since $f$ and $\hat{L}(f(z))$ share the set $\{a(z),-a(z)\} \mathrm{IM}, z_{1}$ is also a zero of $(\hat{L}(f(z))-a(z))(\hat{L}(f(z))+$ $a(z))$ with multiplicity $k_{2}$ (say). Then clearly $z_{1}$ will be a zero of $\alpha(z)$ with multiplicity at least $\min \left\{k_{1}, k_{2}\right\}-1$. Thus no zeros of $f$ and $(f-a(z))(f+a(z))$ are poles of $\alpha(z)$ as long as they are not zeros of $a(z)$. Next proceeding in a similar manner as done in Theorem 1.3, we have (3.6).

Since $f$ and $\hat{L}(f(z))$ are of finite order and share the sets $\{0\}$ CM, so

$$
\frac{\hat{L}(f(z))}{f}=e^{\delta(z)}
$$

where $\delta(z)$ is a polynomial. Now, by Lemma 2.4 we have, $T\left(r, e^{\delta(z)}\right)=S(r, f)$. Since $\hat{L}(f(z)) \not \equiv \pm f$, so $e^{2 \delta(z)} \not \equiv 1$. Clearly,

$$
\begin{aligned}
\bar{N}\left(r, \frac{1}{f^{2}-a^{2}(z)}\right) & \leq N\left(r, \frac{1}{e^{2 \delta(z)}-1}\right)+N\left(r, \frac{1}{a(z)}\right) \\
& \leq 2 T\left(r, e^{\delta(z)}\right)+S(r, f)=S(r, f) .
\end{aligned}
$$

Now, by the Second Fundamental Theorem and using (3.6) and the above fact we can obtain that

$$
T\left(r, f^{2}\right) \leq \bar{N}\left(r, f^{2}\right)+\bar{N}\left(r, \frac{1}{f^{2}}\right)+\bar{N}\left(r, \frac{1}{f^{2}-a^{2}(z)}\right)+S(r, f)=S(r, f) .
$$

Hence we immediately get the conclusion. 
Proof of Theorem 1.19. Since $f$ and $L_{c}^{r} f$ share the sets $\{a(z),-a(z)\}$ CM, so by Lemma 2.5 we have,

$$
\left(L_{c}^{r} f-a(z)\right)\left(L_{c}^{r} f+a(z)\right)=e^{2 \gamma(z)}(f-a(z))(f+a(z)),
$$

where $\gamma(z)$ is a polynomial such that

$$
T\left(r, e^{2 \gamma(z)}\right)=S(r, f) .
$$

Here $f$ is finite order entire function, so by $(2.1), L_{c}^{r} f$ is also finite order entire function. Now, since $f$ and $L_{c}^{r} f$ share the set $\{b(z)\} \mathrm{CM}$, so

$$
\frac{L_{c}^{r} f-b(z)}{f-b(z)}=e^{\eta(z)}
$$

where $\eta(z)$ is a polynomial.

If $e^{\eta(z)} \equiv 1$, then by (3.10) we obtain $L_{c}^{r} f \equiv f$.

Let $e^{\eta(z)} \not \equiv 1$. First we will prove that $m\left(r, \frac{1}{f-b(z)}\right)=S(r, f)$. Let,

$$
R(z, f(z))=\left(L_{c}^{r} f-a(z)\right)\left(L_{c}^{r} f+a(z)\right)-e^{2 \gamma(z)}(f-a(z))(f+a(z)) .
$$

By (3.8) and (3.11) we have, $R(z, f(z)) \equiv 0$. So,

$$
R(z, b(z))=-a^{2}(z)+e^{2 \gamma(z)}\left[a^{2}(z)-b^{2}(z)\right] .
$$

Now our claim is $R(z, b(z)) \not \equiv 0$. On the contrary, suppose that $R(z, b(z)) \equiv 0$. Since $b(z) \not \equiv \pm a(z)$, so $R(z, b(z)) \equiv 0$ implies

$$
e^{2 \gamma(z)} \equiv \frac{a^{2}(z)}{a^{2}(z)-b^{2}(z)} .
$$

Putting this in (3.8) we obtain,

$$
\left(a^{2}(z)-b^{2}(z)\right)\left(L_{c}^{r} f-a(z)\right)\left(L_{c}^{r} f+a(z)\right)=a^{2}(z)(f-a(z))(f+a(z)) .
$$

i.e.,

$$
\left(a^{2}(z)-b^{2}(z)\right)\left(L_{c}^{r} f\right)^{2}=a^{2}(z)(f-b(z))(f+b(z)) .
$$

Suppose $z_{0}$ is a zero of $f-b(z)$. Since $f$ and $L_{c}^{r} f$ share $b(z)$ CM, so $L_{c}^{r} f\left(z_{0}\right)=b\left(z_{0}\right)$. Therefore,

$$
\left(a^{2}\left(z_{0}\right)-b^{2}\left(z_{0}\right)\right) b\left(z_{0}\right)=0 .
$$

Since $b(z) \not \equiv 0$ and $b(z) \not \equiv \pm a(z)$, so either $z_{0}$ is a Picard's exceptional value of $f-b(z)$ or zero of $a(z) \pm b(z)$ or $b(z)$ or both. Therefore,

$$
N\left(r, \frac{1}{f-b(z)}\right)=S(r, f) .
$$

From (3.12), we can observe that if zeros of $f+b(z)$ would not contribute to the zeros of $a^{2}(z)-b^{2}(z)$, then all zeros of $f+b(z)$ will be neutralized by that of $\left(L_{c}^{r} f\right)^{2}$. Thus all the zeros of $f+b(z)$ must be multiple zeros and so we have,

$$
\bar{N}\left(r, \frac{1}{f+b(z)}\right) \leq \frac{1}{2} N\left(r, \frac{1}{f+b(z)}\right) .
$$

By the Second Fundamental Theorem,

$$
\begin{aligned}
T(r, f) & \leq \bar{N}(r, f)+\bar{N}\left(r, \frac{1}{f-b(z)}\right)+\bar{N}\left(r, \frac{1}{f+b(z)}\right)+S(r, f) \\
& \leq \frac{1}{2} N\left(r, \frac{1}{f+b(z)}\right)+S(r, f) \\
& \leq \frac{1}{2} T(r, f)+S(r, f),
\end{aligned}
$$


which is a contradiction. Hence our claim is proved. Therefore by Lemma 2.3, we have

$$
m\left(r, \frac{1}{f-b(z)}\right)=S(r, f) .
$$

Using this from (3.10) and Lemma 2.4 we have,

$$
T\left(r, e^{\eta(z)}\right) \leq m\left(r, \frac{L_{c}^{r} f}{f-b(z)}\right)+m\left(r, \frac{1}{f-b(z)}\right)+S(r, f)=S(r, f) .
$$

From (3.10) we have, $L_{c}^{r} f=f e^{\eta(z)}+b(z)\left(1-e^{\eta(z)}\right)$. Putting this in (3.8) we get,

$$
\begin{aligned}
& \left(e^{2 \eta(z)}-e^{2 \gamma(z)}\right)(f(z))^{2} \\
& =2 b(z) e^{\eta(z)}\left(e^{\eta(z)}-1\right) f-a^{2}(z)\left(e^{2 \gamma(z)}-1\right)-b^{2}(z)\left(e^{\eta(z)}-1\right)^{2} .
\end{aligned}
$$

If $e^{2 \eta(z)}-e^{2 \gamma(z)}=0$, then from (3.14) we get

$$
f=\frac{a^{2}(z)\left(e^{2 \gamma(z)}-1\right)+b^{2}(z)\left(e^{\eta(z)}-1\right)^{2}}{2 b(z) e^{\eta(z)}\left(e^{\eta(z)}-1\right)}
$$

which yields in view of (3.9) and (3.13) that $T(r, f)=S(r, f)$.

If $e^{2 \eta(z)}-e^{2 \gamma(z)} \neq 0$, then using Lemma 2.2, from (3.14) in view of (3.9) and (3.13) we can conclude that,

$$
2 T(r, f)=T(r, f)+S(r, f),
$$

a contradiction. Hence $L_{c}^{r} f \equiv f$.

Acknowledgment. The authors wish to thank the referee for a careful reading and constructive suggestions making the paper readable to more people. The first author is thankful to PRG programme for financial assistance. The second author is thankful to the Council of Scientific and Industrial Research (India) for their financial support under File No: 09/106 (0188)/ 2019- EMR-I.

\section{References}

[1] A. Banerjee and S. Bhattacharyya, Uniqueness of meromorphic functions with their reduced linear c-shift operators sharing two or more values or sets, Adv. Difference Equ. 509, 1-23, 2019.

[2] B. Chen and Z.X. Chen, Entire functions sharing sets of small functions with their difference operators or shifts, Math. Slovaca, 63 (6), 1233-1246, 2013.

[3] N. Cui and Z.X. Chen, The conjecture on unity of meromorphic functions concerning their differences, J. Difference Equ. Appl. 22 (10),1452-1471, 2016.

[4] R.G. Halburd and R.J. Korhonen, Difference analogue of the lemma on the logarithmic derivative with applications to difference equations, J. Math. Anal. Appl. 314, 477487, 2006.

[5] W.K. Hayman, Meromorphic Functions, The Clarendon Press, Oxford 1964.

[6] I. Laine and C.C. Yang, Clunie theorems for difference and q-difference polynomials, J. London Math. Soc. 76 (3), 556-566, 2007.

[7] K. Liu, Meromorphic functions sharing a set with applications to difference equations, J. Math. Anal. Appl. 359, 384-393, 2009.

[8] L. Yang, Value distribution theory, Springer, New York, 1993.

[9] C.C. Yang and H.X. Yi, Uniqueness theory of meromorphic functions, Kluwer Academic Publishers, Dordrecht, 2003. 